\title{
Lapurdum
}

LAPURDUM Euskal ikerketen aldizkaria | Revue d'études basques |

Revista de estudios vascos | Basque studies review

$11 \mid 2006$

Numéro XI

\section{Où on parle (toujours) de génétique textuelle...}

\section{Joana Pochelu}

URL : http://journals.openedition.org/lapurdum/423

DOI : 10.4000/lapurdum.423

ISSN : 1965-0655

Éditeur

IKER

Édition imprimée

Date de publication : 1 novembre 2006

Pagination : 305-309

ISBN : 978-2-86781-474-7

ISSN : $1273-3830$

\section{Référence électronique}

Joana Pochelu, «Où on parle (toujours) de génétique textuelle... », Lapurdum [En ligne], 11 | 2006, mis en ligne le 01 avril 2008, consulté le 19 avril 2019. URL : http://journals.openedition.org/lapurdum/423 ; DOI : 10.4000/lapurdum.423 


\title{
Où on parle (toujours) de génétique textuelle...
}

\author{
Joana POCHELU \\ Doctorante en Études Basques
}

Lors du dernier numéro de Lapurdum ${ }^{1}$, j'écrivais à propos de mon DEA et de la critique génétique.

«Suite au prochain numéro » donc. Je reviens maintenant parler de ma thèse de doctorat, toujours dans le cursus d'Etudes basques à la faculté de Bayonne, sous la direction d'Aurélie Arcocha-Scarcia.

L'étude de cette thèse est d'ordre génétique et sociocritique, et porte sur les manuscrits de travail du roman Soinujolearen semea ${ }^{2}$ de Bernardo Atxaga. Cette approche vise entre autres, à rendre compte de la manière dont se construit un personnage à travers le rapport de la réalité et du texte, ou à montrer comment l'incipit se met en place dans ce roman ancrée dans l'historicité et la fiction, ou encore, à illustrer comment l'auteur est appréhendé à partir du texte, comme un des éléments de la textualité.

Le dossier génétique que j'ai constitué, contient des centaines de manuscrits de travail de ce roman, et plus spécifiquement des manuscrits informatisés (ou tapuscrits), et se consacre strictement à cet état d'écriture sur ordinateur. C'est pour plusieurs raisons que je limite ce corpus au format informatique.

C'est d'abord une question matérielle qui m'a poussée à me concentrer sur les seuls tapuscrits : Atxaga m'a fait part de la difficulté pour lui de retrouver les éléments qui concernent ce roman, car tout est dispersé dans plusieurs cahiers, carnets, papiers, et autres documents qui ont servi pour son travail d'écriture, et dont il se sert toujours. Il note une idée ou une remarque sur un carnet, lors d'une promenade par exemple, et peut les utiliser seulement quelques années après quand il aura trouvé l'inspiration pour s'en servir. Il ne pouvait donc pas se séparer physiquement de ces papiers, ni même en faire des copies, car il ne sait pas exactement ce qu'il a et où il l'a. Ce ne serait alors pas une étude précise car je n'aurais pas à ma disposition tout ce qui a été la genèse de ce travail.

1 - «A propos de mon DEA. Où on parle de génétique textuelle... » (p. 331-336), Lapurdum X, 2005, Bayonne, Centre de Recherche sur la Langue et les Textes Basques IKER-UMR 5478.

2 - B. Atxaga, Soinujolearen semea, Iruñea, Pamiela, 2003. 
Et j'en arrive là à une autre des raisons, et non des moindres, pour laquelle je fixe ma recherche aux manuscrits informatisés : j'atteins par ce fait une exhaustivité. En effet, je sais de façon sûre et certaine que je suis en possession de tout l'état informatique de l'avant-texte, car l'écrivain m'a remis absolument tout ce qu'il avait gardé sur son ordinateur, de l'écriture de Soinujolearen semea. Raymonde DebrayGenette cite Claudine Gothot-Mersch qui écrit que "l'exhaustivité se révèle aussi impossible qu'elle est indispensable [et que] la génétique est le règne du "toutefois" " ${ }^{3}$. Indispensable, indiscutablement, et dans notre cas elle se révèle possible et même réelle.

Enfin, une dernière raison est mon intention de montrer que la critique génétique ne s'arrête pas au seuil de la chose manuscrite. Comme le dit si bien Philippe Willemart ${ }^{4}$, « la question classique que nous entendons invariablement de tous ceux qui découvrent la critique génétique et qui veulent souvent nous prendre au piège est : Que va devenir votre approche à l'ère de l'informatique? " Je voudrais donc démontrer que les écrits sur ordinateur valent tout autant pour une étude de genèse ${ }^{5}$. L'ordinateur n'est pas la fin de la critique génétique !

Willemart écrivait cela à propos de Dragon Naturally Speaking, qui est un logiciel permettant de dicter directement à l'ordinateur ce que l'on veut écrire sans passer par la phase de saisie du texte. Ainsi, l'usager dicte à la machine, tout en regardant ce qui s'écrit, relit, et peut donc procéder à un ajout, « tout en faisant disparaitre le mot remplacé, le paragraphe transposé et ainsi toute trace génétique par la commande « effacer ça ». Dans ces conditions-là, afin d'avoir encore des brouillons, il ne faudrait apporter aucune correction ni changement à la première version que l'auteur sauvegarderait, puis il travaillerait sur une copie de cette version et ainsi de suite, pour laisser des traces au généticien.

Mais sans aller jusqu'à l'éventualité (extrême) de l'utilisation par les écrivains contemporains de ce logiciel-là, restons en au fait de l'écriture par ordinateur qui a remplacé celle sur papier, et reposons donc la question : sur quoi les généticiens peuvent-ils travailler à l'époque de l'ordinateur et du traitement de texte par informatique?

Ce à quoi je réponds : l'œuvre qui a été écrite sur ordinateur n'a t'elle donc pas connu aussi un processus de création ? Est-ce donc l'ordinateur lui-même qui l'a écrit, et d'un seul jet, ou est-ce qu'il y a eu élaboration de la part de l'écrivain?

Il est évident que même sur ordinateur l'œuvre a une origine et, par conséquent, une étude génétique est donc faisable.

Le généticien sera confronté sans doute à tel ou tel type de documents selon qu'il s'agisse de la méthode de travail d'un auteur ou d'un autre. En effet, les tapuscrits

3 - R. Debray-Genette, «Du bon usage de l'exhaustif : l'Hérodias de Flaubert édité par G. Bonaccorso » in Genesis n³, éd Jean-Michel Place, 1993, p. 157-164.

4 - Ph. Willemart, "La critique génétique face au logiciel de reconnaissance vocale », in Genesis n²3, éd Jean-Michel Place, 2004, p. 161-165.

5 - Au moment même où cet article est en impression, vient de paraître le Genesis n ${ }^{\circ} 27$ dont les chroniques sont regroupées sous le titre « Le généticien et l'ordinateur». 
qu'il aura à étudier dépendront de l'écrivain qui aura enregistré chaque étape de sa création, ou pas. Puis cela dépendra aussi du type de document que l'auteur produit, s'il écrit d'abord des notes sur papiers pour les rédiger ensuite sur son ordinateur, ou si la phase de notes s'y fait directement, si on trouve quelques bouts d'écriture isolés, des chapitres abandonnés, des versions successives, etc. Mais n'est-ce pas aussi exactement le même cas avec les écrivains qui écrivent sur papiers ? La seule différence serait que les tapuscrits pourraient contenir plusieurs états, et qu'elles ne soient pas repérables si chacune d'entre-elles n'a pas été sauvegardée.

Toujours est-il que les nouvelles technologies sont d'une avancée si rapide, que si les habitudes d'écriture et de créations changent de support, les outils pour l'étude de ces mêmes supports connaissent eux aussi une évolution si non parallèle, immédiate. Ceci, non pas par souci « de céder à un effet de mode ou au désir d'égayer la poussière de nos archives de quelques paillettes high-tech, mais bien de prendre en compte un enjeu capital pour la génétique ${ }^{6}{ }^{6}$

C'est justement dans le but d'" aider les philologues et, plus particulièrement pour faciliter l'étude préalable à toute interprétation de génétique textuelle », qu'a été conçue MEDITE (Machine d'Étude Diachronique et Interprétative du Travail de l'Écrivain) ${ }^{7}$. Il s'agit d'importer dans ce logiciel un dossier constitué de différentes versions, pour ensuite " comparer deux états de textes littéraires en indiquant les transformations textuelles opérées de l'un à l'autre [...] ». "C'est un programme qui repère automatiquement la séquence temporelle des corrections, adjonctions, et ratures opérées par l'auteur », faisant passer d'un état à un autre d'un même texte.

L'interprétation génétique est en effet facilitée par cette machine qui effectue le travail préliminaire, et dont l'interface permet la visualisation immédiate de tout ce qui se passe entre deux versions d'un même texte, grâce à des surlignements de couleurs différentes selon le type de modification. De surcroît, "l'exhaustivité des transformations nous est [textuellement] donnée, dans un tableau inférieur ». Le travail fait donc ici «l'objet d'une étude systématique».

On comprendra que «le logiciel MEDITE constitue pour la critique génétique un outil nécessaire. Nécessaire pour la rapidité des résultats qui est un gain de temps et de travail pour le généticien, nécessaire aussi pour une scientificité plus sûre, nécessaire pour un usage qualitatif des données quantitatives. »

Cela me permettra en effet, de travailler sur un nombre considérable de données et de procéder également, au besoin, à des études statistiques, pour caractériser le type de changements opérés par Atxaga. Un autre outil alliant littérature et informatique m'aidera également à l'heure de comparer les statistiques ressorties des tapuscrits à ceux du texte définitif. En effet, le programme $\mathrm{EPG}^{8}$ (Ereduzko Prosa Gaur, le Modèle de la Prose Aujourd'hui), disponible sur Internet ${ }^{9}$, offre la possibilité de consulter à quel endroit, avec quelle occurrence et dans quel contexte

6 - J-L. Lebrave, « Hypertextes - Mémoires - Écriture », in Genesis n 5, éd Jean-Michel Place, 1994, p.9-24. 7 - J. G. Ganascia, I. Fenoglio, J-L. Lebrave, Manuscrits, genèse et documents numérisés. EDITE : une étude informatisée du travail de l'écrivain, Document numérique, numéro spécial sur le thème «temps et document, 2004.

8 - J-L. Zabala, «Ereduzko idazleak kopiatzeko aukera » (l'occasion de copier les écrivains modèles), in Berria, 20-11-2003. Ce programme a été crée pour que l'utilisateur puisse vérifier comment les écrivains emploient le mot sur lequel il doute. Mais en ce qui me concerne, je l'utilise plutôt pour retrouver un passage du roman dense Soinujolearen Semea plus rapidement.

9 - http://www.ehu.es/euskara-orria/euskara/ereduzkoa/ 
est utilisé tel mot ou telle expression dans telle œuvre de tel écrivain. Et par chance, Soinujolearen semea se trouve dans le corpus de ce programme en chantier.

Pour pouvoir les comparer grâce au logiciel MEDITE, le généticien, lorsqu'il se trouve en face de plusieurs états ou versions d'un même texte qui sont sous forme manuscrite, doit au préalable, les transcrire linéairement, afin d'avoir ces feuillets sous forme électronique. En ce qui nous concerne, nous avons fait l'économie de cette étape ardue du travail, car, vous l'aurez maintenant compris, les manuscrits de ce dossier génétique étaient déjà dactylographiés.

Mais cette analyse des tapuscrits par le logiciel MEDITE ne sera que le travail préliminaire de l'étude de la genèse de Soinujolearen Semea. Je vais par la suite, interpréter ces données à travers une lecture sociocritique ${ }^{10}$.

Pourquoi procéder à l'interprétation génétique à travers une approche sociocritique ? Parce que c'est le texte que la sociocritique prend comme point de départ. Et cette perspective concorde totalement à mon approche d'Atxaga qui porte sur l'œuvre d'Atxaga puisque, le texte est au centre du projet sociocritique et que l'écrivain en tant que tel, n'est pas le sujet mais l'objet de l'écriture. C'est au texte (en devenir) que s'intéresse mon étude.

En outre, la sociocritique a plusieurs avantages. Elle permet de dépasser une lecture formaliste pour qui le texte est autosuffisant, et qui empêche de rendre compte de toutes les possibilités de celui-ci. Aussi, elle « empêche la fascination érudite pour le contexte, qui s'exerce toujours au détriment du texte lui-même ${ }^{11}$.

Claude Duchet ${ }^{12}$ dit que "la génétique se situe très exactement à cette articulation entre l'écriture privée et l'écrit publié. Et l'hypothèse de la sociocritique est que cette écriture "privée » persiste toujours [...] dans l'écrit rendu public. »

«Tout ce qu'on peut écrire [...] habite le texte publié, comme trace indélébile de virtualités, de possibles refusés, écartés, effacés, censurés, différés ou déplacés, mais aussi comme marques d'adhérence à la totalité sociale à laquelle le texte participe toujours. »

Cette étude de génétique et de sociocritique, en s'appuyant sur un corpus électronique, a comme projet de démontrer que l'ordinateur n'est pas la fin de la génétique, mais que bien au contraire, c'est un outil qui permet d'aider l'étude des textes littéraires.

J'ose espérer que de surcroît, cette thèse contribuera à l'avancement de la recherche en littérature d'expression basque, qui est à temps de ne pas rater le train en se mettant à jour en matière de critique génétique et d'outil informatique. Certes, au Pays Basque nous avons déjà constaté quelques balbutiements dans le domaine, et Jon Casenave remarquait d'ailleurs un "signe précurseur d'une

10 - Mot crée par Claude Duchet en 1971.

11 - P. Laforgue, Balzac dans le texte : Études de génétique et de sociocritique, Saint-Cyr-Sur-Loire, Christian Pirot, 2006

12 - Cl. Duchet, « Sociocritique et génétique (Entretien avec Anne Herschberg Pierrot et Jacques Neefs)», in Genesis n6, éd Jean-Michel Place, 1994, p. 117-127. 
évolution en matière d'édition et de socialisation du texte et de l'œuvre littéraires ", ceci, lors de la divulgation du manuscrit en langue basque du XVI ${ }^{\mathrm{e}}$ siècle rédigé par Juan Perez Lazarraga ${ }^{13}$. En fait, cet événement fut « inédit dans l'histoire de la littérature basque, [car] le grand public a pris connaissance et a disposé d'un document d'exception en même temps que la communauté savante, [puisque] l'apparition du texte original sur Internet et en CD-ROM [se faisait] le jour même de la conférence de presse qui a fait connaitre la nouvelle. »

La machine informatique est entrée dans notre société, et s'est désormais établie dans nos habitudes de tous les jours, dès lors, et avec d'autant plus de raisons, la recherche littéraire ne doit pas avoir peur de ces nouvelles technologies, mais à l'inverse devrait prendre le train en marche. Et c'est donc ce que je vais tenter de faire à travers cette thèse.

\section{Bibliographie}

- Duchet, $\mathrm{Cl}$., « Sociocritique et génétique (Entretien avec Anne Herschberg Pierrot et Jacques Neefs) », in Genesis n6, éd Jean-Michel Place, 1994, p. 117-127.

- Ganascia, J. G., Fenoglio, I., Lebrave, J.-L., Manuscrits, genèse et documents numérisés. EDITE : une étude informatisée du travail de l'écrivain, Document numérique, numéro spécial sur le thème «temps et document, 2004.

- Laforgue, P., Balzac dans le texte : Études de génétique et de sociocritique, SaintCyr-Sur-Loire, Christian Pirot éd., 2006.

— Willemart, Ph., «La critique génétique face au logiciel de reconnaissance vocale », in Genesis n²3, éd Jean-Michel Place, 2004, p. 161-165.

13 - J. Casenave, «A l'occasion de la mise en ligne du manuscrit Lazarraga : éléments de réflexion sur la conversion numérique des corpus littéraires du domaine basque », in Lapurdum VIII, Bayonne, Centre de Recherche sur la Langue et les Textes Basques IKER - UMR 5478, 2003, p. 97-121.

Voir aussi du même auteur, "La revitalisation numérique du patrimoine littéraire territorialisé » Article écrit en collaboration avec trois informaticiens du LIUPPA (Laboratoire d'informatique de l'Université de Pau et des Pays de l'Adour), Ch. Marquesuzaa, P. Dagorret et Mauro Gaio, Colloque international EBSIENSSIB, Montréal, Octobre 2004. Publication des Actes courant 2005. 